\title{
Preconditioning of Human Adipose-derived Stromal/Stem Cells: Evaluation of Short-term Preincubation Regimens to Enhance their Regenerative Potential
}

\author{
Patrick C Baer ${ }^{1 *}$, Jürgen M Overath ${ }^{1}$, Anja Urbschat ${ }^{2}$, Ralf Schubert ${ }^{3}$ and Helmut Geiger ${ }^{1}$ \\ ${ }^{1}$ Division of Nephrology, Department of Internal Medicine III, Goethe-University, 60590 Frankfurt/M., Germany \\ ${ }^{2}$ Department of Urology and Pediatric Urology, University Hospital Marburg, Philipps-University, 35043 Marburg, Germany \\ ${ }^{3}$ Department of Paediatrics, Goethe-University, 60590 Frankfurt/M., Germany
}

\begin{abstract}
Objective: Stem cell-based therapy seems to be a promising option to support regeneration after organ failure and tissue injury. The transplantation of MSCs requires cells with a maximum regenerative capacity. Therefore, the research on new strategies to improve the release of regenerative factors of MSCs is urgently needed.

Methods: Human adipose-derived stromal/stem cells (ASC) were isolated from lipoaspirates, characterized, and cultured. Cells were either cultured under standard conditions or preconditioned by incubation in a hypoxic environment $\left(0.5 \% \mathrm{O}_{2}\right)$ or in normoxia in the presence of recombinant human tumor necrosis factor- $\alpha$ (TNF $\alpha$ ) or recombinant human epidermal growth factor (EGF) for 48 hours. First, seven selected regeneration promoting factors were evaluated by GPCR analysis. Afterwards the secretome of ASCs was estimated using a commercially available protein array for 507 proteins.
\end{abstract}

Results: PCR analysis showed a differential induction of ASCs' gene expression by the three pretreatments. Whereas ASCs in hypoxia showed a significant mRNA induction of VEGF, FGF-7, and IGF-II, the other pretreatments induced no significant change in VEGF expression. The gene expression of HB-EGF and M-CSF was significantly induced in hypoxia and by incubation with TNFa, but not EGF. Angiopoietin-like 1 mRNA was not significantly induced by all three preconditioning regimens. Evaluation by the protein array revealed that from the 507 proteins investigated $21.9 \%$ were found to be more than five-fold increased after incubation in hypoxia (111 of 507 proteins). Preincubation with EGF resulted in an upregulation of $32.3 \%$ (164/507), whereas TNFa upregulated $28.8 \%$ of all proteins evaluated $(146 / 507)$.

Conclusion: The findings indicate that all three preconditioning regimens induced a wide variety of proteins. However, short-term pretreatment with EGF induced the highest quantity of proteins, and, therefore, appears to be the best preconditioning regime for cell therapeutic approaches.

Keywords: Preconditioning; Pretreatment; Stem cells; Medium; Regeneration; Hypoxia; Mesenchymal stromal/stem cells

Abbreviations: ASCs: Adipose-derived Mesenchymal Stromal/ Stem Cells; EGF: Epidermal Growth Factor; FGF-7: Fibroblast Growth Factor-7; HB-EGF: Heparin-Binding EGF-like Growth Factor; Hyp: Preconditioning in a Hypoxic Environment; IGF-II: Insulin-like Growth Factor-II; M-CSF: Macrophage Colony-Stimulating Factor; MIP-1: Macrophage Inflammatory Protein 1; MSCs: Mesenchymal Stromal/Stem Cells; qPCR: quantitative Real Time Polymerase Chain Reaction; TNFa: Tumor Necrosis Factor-alpha; VEGF: Vascular Endothelial Growth Factor

\section{Introduction}

Stem cell-based therapy seems to be a promising option to support regeneration after organ failure and tissue injury. The organprotective effects of mesenchymal stromal/stem cells (MSCs) and their conditioned medium (CM) have been investigated in the last decade, demonstrating that either transplanted cells or their CM promote regeneration after several organ- and tissue injuries. The transplantation of MSCs requires cells with a maximum regenerative capacity. Therefore, the development of new strategies to improve the release of regenerative factors of MSCs is urgently needed. In vitro pretreatment regimens able to optimize the regenerative capacity of stem cells should be in the focus of further studies aiming to enhance the regeneration process. During the last years, optimization of the beneficial effects of cell therapy has been investigated, seeking to enhance survival, engraftment, and paracrine properties of MSCs [1] In this case, various in vitro pretreatment strategies ("preconditioning") have been recently applied to enhance the regenerative capacity of MSCs [2-4]. MSC pretreatment may include exposure of cells to physiological stimuli such as cytokines, growth factors, biophysical stimuli, heat shock, pharmacological agents, cell-cell-contacts, or hypoxia [1-4]. Preconditioning by hypoxia has been shown to initiate the secretion of regenerative factors and to enhance cell survival $[5,6]$. Findings of others suggest that hypoxic preincubation of MSCs for two days induces metabolic changes that yield higher in vivo cell retention after transplantation [6]. Therefore, choosing appropriate pretreatment regimens may provide a simple but effective way of promoting survival

*Corresponding author: Patrick C Baer, M.D, Ph.D, Johann Wolfgang Goethe University, Department of Internal Medicine III, Division of Nephrology, TheodorStern-Kai 7, 60590 Frankfurt/M., Germany, Tel: 0049/69/6301-5554; Fax: 0049/69/6301-4749; E-mail: patrick.baer@kgu.de

Received February 29, 2016; Accepted March 12, 2016; Published March 19 2016

Citation: Baer PC, Overath JM, Urbschat A, Schubert R, Geiger H (2016) Preconditioning of Human Adipose-derived Stromal/Stem Cells: Evaluation of Short-term Preincubation Regimens to Enhance their Regenerative Potential. J Stem Cell Res Ther 6: 331. doi:10.4172/2157-7633.1000331

Copyright: ( 2016 Baer PC, et al. This is an open-access article distributed unde the terms of the Creative Commons Attribution License, which permits unrestricted use, distribution, and reproduction in any medium, provided the original author and source are credited. 
and migration to the site of injury, enhancing regenerative properties, and boosting the regenerative capability of transplanted stem cells in cell therapy approaches [1].

In general, MSCs have been identified throughout the whole body as immature cells. Classically they were isolated from the bonemarrow, but also from nearly all adult tissues (e.g. adipose tissue) and solid organs (e.g. liver, kidney) [7-9]. MSCs represent a rare population (or populations) in the perivascular niche (or are derived from perivascular cells or pericytes [10]) within fully specialized tissues. MSCs release a number of regeneration promoting, pro-angiogenic, anti-inflammatory and immune-modulatory factors that improve regeneration in injured organs or tissue [11]. Although numerous studies using MSCs (or their CM) as potential therapeutic agents have been published, our understanding is incomplete regarding how this regenerative activity is governed by interactions with resident cells, growth factors and cytokines.

The present study investigates the potential of different preconditioning regimens to enhance the release of factors, and, therefore, the regenerative potential of human adipose-derived MSCs (ASCs). We firstly evaluated the effects of three different preconditioning regimens on seven selected regeneration promoting factors by quantitative real-time polymerase chain reaction (qPCR) analysis. Afterwards we identified the release of 507 proteins into the cell culture medium using a commercially available protein array.

\section{Methods}

\section{Cell isolation and culture}

Human adipose-derived adult mesenchymal stromal/stem cells (ASC) were isolated from lipoaspirates from seven female donors undergoing cosmetic liposuction in accordance to the local ethical committee. Aspirated tissue was digested at $37^{\circ} \mathrm{C}$ with $0.075 \%$ collagenase I (Biochrom, Berlin, Germany) under continuous agitation for 45 minutes. The stromal-vascular fraction was separated from the remaining fibrous material and the floating adipocytes by centrifugation at $300 \mathrm{~g}$. The sedimented cells were washed with PBS and filtered through a $100 \mu \mathrm{m}$ pore filter (Millipore, Schwalbach, Germany). Erythrocyte contamination was reduced by density gradient centrifugation with Bicoll (Biochrom, Berlin, Germany). Finally, cells were plated for initial cell culture, and cultured at $37^{\circ} \mathrm{C}$ in an atmosphere of $5 \% \mathrm{CO}_{2}$ in humid air. Primary cell isolates and cultured cells were fully characterized, as described previously $[12,13]$.

Dulbecco's modified Eagle's medium (DMEM; Sigma, Taufkirchen, Germany) was used with a physiologic glucose concentration (100 $\mathrm{mg} / \mathrm{dl}$ ) supplemented with $10 \%$ fetal calf serum (FCS; PAA, Cölbe, Germany) as the culture medium. The medium was replaced every three days. Subconfluent cells (85-90\% confluency) were passaged by trypsinization. The $1^{\text {st }}-4^{\text {th }}$ passage of ASC from 7 different female donors was used for the experiments. Cell morphology was examined by phase contrast microscopy. Expression of characteristic markers was proven by immunofluorescence staining for CD90 expression and flow cytometric analysis of CD73, CD90, and CD105 expression, as described previously $[13,14]$. Tri-lineage differentiation potential of cultured ASC was proven by specific media, as described previously $[14,15]$.

\section{Preconditioning regimen and conditioned medium}

Cells were either cultured under standard conditions (controls in normoxia $\left(21 \% \mathrm{O}_{2}\right)$ ) or preconditioned by incubation in a hypoxic environment $\left(0.5 \% \mathrm{O}_{2}\right)$ or in normoxia supplemented with recombinant human tumor necrosis factor- $\alpha$ (TNFa Immunotools, Friesoythe, Germany No. 11343015) or recombinant human epidermal growth factor (EGF, Immunotools, Friesoythe, Germany No. 11343406). All treatments were performed for $48 \mathrm{~h}$ in serum-free low-glucose DMEM. For this purpose, cells were grown to subconfluency and washed twice with PBS. Then, TNFa or EGF was added to fresh serum-free DMEM in a final concentration of $10 \mathrm{ng} / \mathrm{ml}$. Cells treated with hypoxia received fresh serum-free DMEM without supplements and were placed in an in vivo $\mathrm{O}_{2} 400$ (Baker and Baker Ruskinn, Sanford, USA) at $0.5 \%$ oxygen. After 48 hours, the medium was removed, centrifuged at 1,000 $\mathrm{g}$ for $10 \mathrm{~min}$ and processed as described below or stored at $-80^{\circ} \mathrm{C}$ for further processing. Furthermore, cell viability after preconditioning was determined by a photometric assay using 2,3-Bis-(2-Methoxy4-Nitro-5-Sulfophenyl)-2H-Tetrazolium-5-Carboxanilide (XTT), as described previously [16]. In brief, subconfluent cells in 96-well plates were preconditioned for 48 hours as described above. Afterwards, XTT reagent was added to wells as described by the manufacturer (Applichem, Darmstadt, Germany) and incubated at $37^{\circ} \mathrm{C}$. Absorbance was measured in a microplate reader at $490 \mathrm{~nm}$ vs. $650 \mathrm{~nm}$.

\section{Quantitative Real Time PCR (qPCR)}

Total cellular RNA was isolated immediately after preconditioning. RNA extraction was performed using the single-step RNA isolation from cultured cells by a modified protocol of Chomczynski and Sacchi [17]. After RNA extraction and synthesis of cDNA, quantitative PCR was carried out using a SYBR Green Rox Mix with the following conditions: $15 \mathrm{~min}$ at $95^{\circ} \mathrm{C}$ for enzyme activation and 35 cycles of 15 $\mathrm{s}$ at $95^{\circ} \mathrm{C}$ for denaturation, $30 \mathrm{~s}$ at $63^{\circ} \mathrm{C}$ for annealing, $30 \mathrm{~s}$ at $72^{\circ} \mathrm{C}$ for elongation, and $4^{\circ} \mathrm{C}$ for $5 \mathrm{~min}$. Reactions were carried out in duplicate in 96 well plates. Quantification of the PCR fragment was carried out using the Eppendorf realplex ${ }^{2}$ Mastercycler ep gradient S (Eppendorf, Hamburg, Germany). Melting curve analysis was performed and the mean cycle threshold $(\mathrm{Ct})$ values were used to calculate gene expression levels. Relative quantification was estimated by the $\Delta \Delta \mathrm{C}_{\mathrm{T}}$ method [18] with normalization to $\beta$-actin. The relative mRNA expression of the target gene was calculated by using $2^{-\triangle \Delta C T}$. PCR products were verified on an agarose gel electrophoresis. Primer pairs were synthesized by Invitrogen (Karlsruhe, Germany) and are listed in Table 1.

\section{Protein array}

We used a commercially available protein array (Tebu-Bio, Offenbach, Germany, Human Antibody Array No. AAH-BLG-1-4) for the simultaneous detection of the relative expression of 507 human proteins in the supernatant. In brief, all supernatants were collected and centrifuged for $10 \mathrm{~min}$ at $1,000 \mathrm{~g}$. Then, the protein content of the supernatants was determined by a routine assay using bicinchoninic acid (BCA). Supernatants were concentrated using a 3 $\mathrm{kDa}$ molecular weight cut-off Amicon Ultra-4 filter (No. UFC800324, Merck Millipore, Darmstadt, Deutschland) for $24 \mathrm{~min}$ at 3,060 g. The protein content of the supernatants was again determined by the BCA assay. Finally, the protein array was exactly processed according to the manufacturer's protocol. The readily prepared array was then sent to the manufacturer, which performed the measurement of the slides and normalization of the measured data (positive control normalization without background).

\section{Statistical analysis}

The data were expressed as mean \pm SD. The comparison between 


\begin{tabular}{|c|c|c|c|c|}
\hline Gene & Primer forward & Primer reverse & Product length & NCBI Reference \\
\hline VEGF & CTGTCTAATGCCCTGGAG & TATCGATCGTTCTGTATCAGT & $268 \mathrm{bp}$ & NM_001025366 \\
\hline HB-EGF & AAGAGGGACCCATGTCTTCG & GATTTTCCACTGGGAGGCTCA & $148 \mathrm{bp}$ & NM_001945 \\
\hline IGF-II & GATTAATTACACGCTTTCTGT & CTGTTGTATCAAGGATAGAGG & $165 \mathrm{bp}$ & NM_000612 \\
\hline Angiopoietin-like 1 & GTTATTCAGAAAAGAACAGACG & TTTTATCACTCCAGTCTTCTAAT & $175 \mathrm{bp}$ & NM_004673 \\
\hline MIP-1a & TCAGACTTCAGAAGGACAC & TAGTCAGCTATGAAATTCTGTG & $243 b p$ & NM_002983 \\
\hline FGF-7 & CCCTGAGCGACACACAAGAA & TTCCACCCСTTTGATTGCCA & $199 \mathrm{bp}$ & NM_002009 \\
\hline M-CSF & GCAGCTGCAGGAACTCTCTT & TGGTCACCACATCTTGGCTG & $227 \mathrm{bp}$ & NM_000757 \\
\hline$\beta$-Actin & ACTGGAACGGTGAAGGTGAC & AGAGAAGTGGGGTGGCTTTT & $169 \mathrm{bp}$ & NM_001101 \\
\hline
\end{tabular}

Table 1: Primers used for PCR.
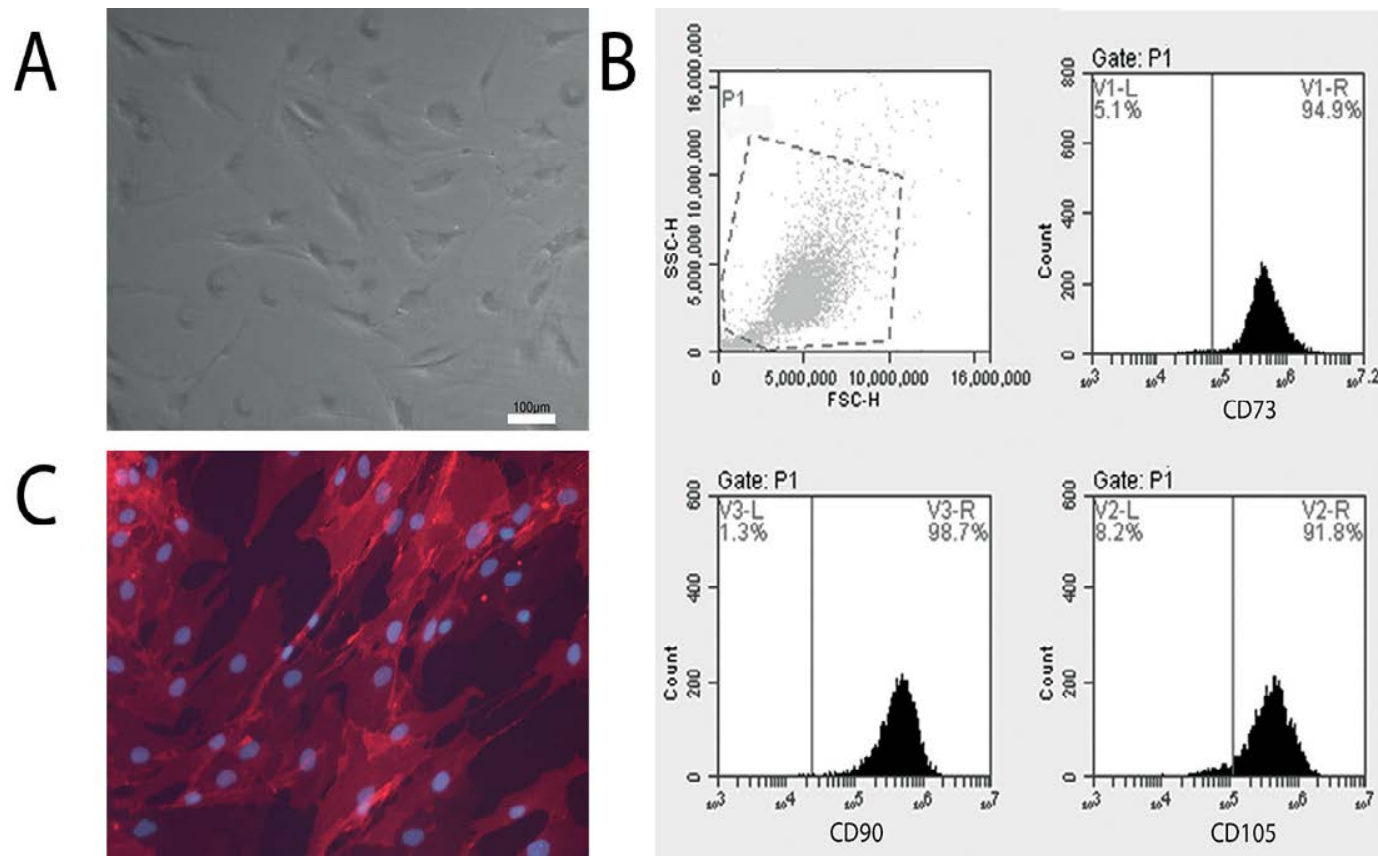

(A) Characteristic phase contrast microscopy of human adipose-derived MSCs (ASCs) cultured in standard cell culture (bar: 100 um). (B) Representative flow cytometric histograms of the expression of characteristic markers CD73, CD90, and CD105. Line shows discrimination of negative cells (isotype controls). (C) Characteristic immunofluorescence staining of CD90 expression (Nuclei were stained with DAPI).

Figure 1: Cell characterization.

groups was performed by one-way analysis of variance (ANOVA) and Bonferroni post-hoc test using Prism 5 software (GraphPad). p values $<0.05$ were considered significant.

\section{Results}

\section{Characterization of human adipose-derived mesenchymal stromal/stem cells (ASCs)}

Cell morphology was evaluated by phase contrast microscopy. The cells displayed a spindle-shaped fibroblastoid morphology in culture (Figure 1A). Expression of characteristic markers for mesenchymal stromal/stem cells (CD73, CD90, CD105) was shown by flow cytometric analysis (Figure 1B). A comprehensive characterization of ASCs has been shown earlier [13]. In addition, expression of CD90 was shown by immunofluorescence staining (Figure 1C).

\section{Effect of preconditioning regimens on cell viability}

Cell viability after the preconditioning regimens was determined by XTT assay. The XTT assay is a colorimetric assay used to determine cell viability as a function of cell number based on metabolic activity of the cultured cells. We detected no significant differences in cell viability after the pretreatments compared to the control (Figure 2).

\section{Evaluation of preconditioning by $\mathrm{QPCR}$}

We investigated the potency of three different preconditioning regimens on the gene expression of ASCs. Therefore, we analysed the mRNA of seven selected potential regeneration promoting factors (VEGF, FGF-7, HB-EGF, IGF-II, Angiopoietin-like 1, MIP-1 (CCL3), $\mathrm{M}-\mathrm{CSF}$ ) by qPCR analysis (Figure 3 ). The qPCR results highlight the differential induction of ASCs' gene expression by thosee three pretreatments. Whereas the Hyp treated ASCs showed a significant VEGF mRNA induction, the other preincubation regimens induced no significant changes in the VEGF mRNA expression. Also FGF-7 and IGF-II mRNA were only induced by the Hyp. Angiopoietin-like 1 mRNA was not significantly induced by all three preconditioning regimens. The gene expression of the other two factors (HB-EGF, M-CSF) was significantly induced by incubation with TNFa or by a Hyp. Interestingly, none of the evaluated mRNAs was induced after incubation with EGF at the chosen endpoint $(48 \mathrm{~h})$. Nevertheless, in regard of the results of the protein array it should be mentioned that 


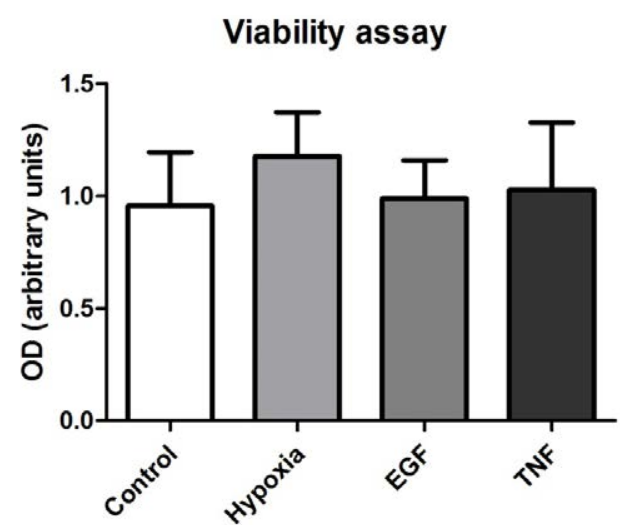

ASCs from three donors were cultured in 96 well plates (each in quintuplicate) and preconditioned for 48 hours (as indicated). Then, the XTT assay was performed and optical density (OD) was measured in a microplate reader at $490 \mathrm{~nm}$ vs. $650 \mathrm{~nm}$. Results are shown as arbitrary units (mean \pm SD). No significant effects on the cell viability could be detected.

Figure 2: Viability assay.

none of the seven factors was de novo induced by the preincubation methods. All factors were constitutively expressed by ASCs - as proven by the $\mathrm{Ct}$ value and the melting curve analysis of the qPCR data (data not shown).

\section{Evaluation of preconditioning by protein array}

Based on the hypothesis that factors released from ASCs represent a key mechanism to enhance organ and tissue regeneration after injury, we evaluated whether the preconditioning regimens increase the release of proteins into the culture supernatant. Herefore, we used a commercially available protein array for the simultaneous detection of 507 human proteins in the supernatant of the preconditioned ASCs in comparison to ASCs cultured under standard conditions (control). The data from this array were evaluated, whereas only values (arbitrary units) which increased more than five-fold compared to the control were regarded as induced (Figure 4). Furthermore, we did not use stimulation values $<500$. From the 507 proteins in the assay, $21.9 \%$ were found to be more than five-fold increased after incubation in a Hyp (111 of 507 proteins) (Figure 4). Preincubation with EGF resulted in an upregulation of $32.3 \%$ (164/507), whereas TNFa upregulated $28.8 \%$ of all proteins evaluated (146/507) (Figure 4). Complete results from all 507 proteins and the internal controls are shown in a supplemental online table (Table S1).

Compared to the seven selected regeneration promoting factors evaluated by PCR analysis, the results of the protein array are not completely consistent. Interestingly, the amount of protein release of all seven factors was induced by each pretreatment regimen, whereas qPCR analysis revealed a differential induction of the mRNA expression. For example the amount of Angiopoietin-like 1 protein in the cell supernatant exhibits a more than five-fold induction by each preincubation regimen, whereas no significant induction of the mRNA expression could be detected. On the other hand, HB-EGF protein was significantly enhanced in the supernatant by all treatments similarly to the mRNA expression (Hyp and TNFa significant, EGF by trend). It should also be mentioned that similar to the mRNA none of the proteins was de novo induced by the preincubation methods. All seven proteins were constitutively expressed in non-pretreated ASCs, as proven by the protein array (Supplemental Table S1).

\section{Discussion}

Adult adipose-derived stromal/stem cells are multipotent cells with strong paracrine activities based on the release of various regeneration promoting factors [19]. Therefore, ASCs are promising cells for regenerative medicine and cell therapy. In this regard, studies have demonstrated that transplantation of MSCs exerts beneficial effects,
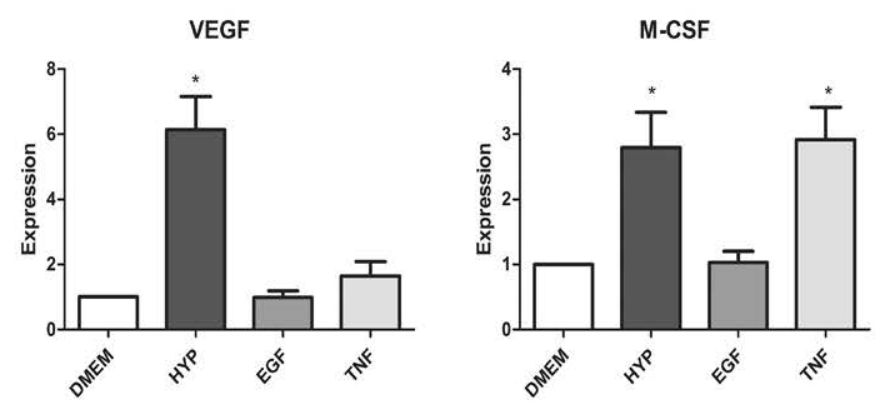

HB-EGF

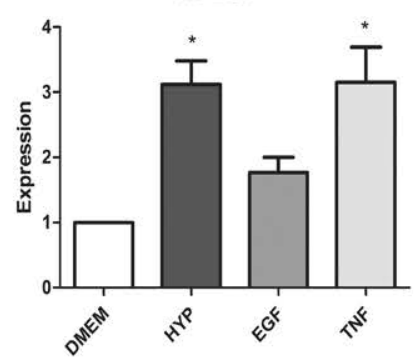

FGF-7

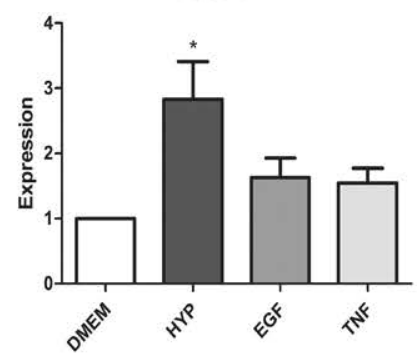

MIP-1

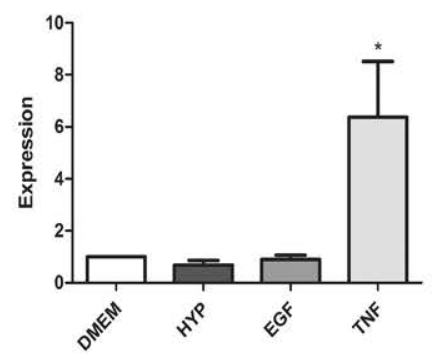

Angiopoietin-like 1

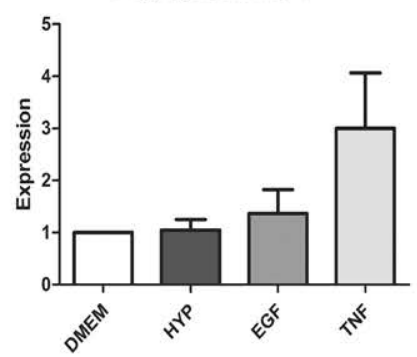

Expression was measured in total RNA from ASCs after preconditioning regimens. The expression levels in each experiment were normalized to a housekeeping gene $\left(\beta\right.$-actin) and are expressed relative to the control $\left(2^{-\Delta \triangle C T}\right) .{ }^{*} p<0.05$ versus control, $n=5-7$.

Figure 3: Effect of preconditioning on gene expression of selected factors. 
Citation: Baer PC, Overath JM, Urbschat A, Schubert R, Geiger H (2016) Preconditioning of Human Adipose-derived Stromal/Stem Cells: Evaluation of Short-term Preincubation Regimens to Enhance their Regenerative Potential. J Stem Cell Res Ther 6: 331. doi:10.4172/2157-7633.1000331

Page 5 of 7

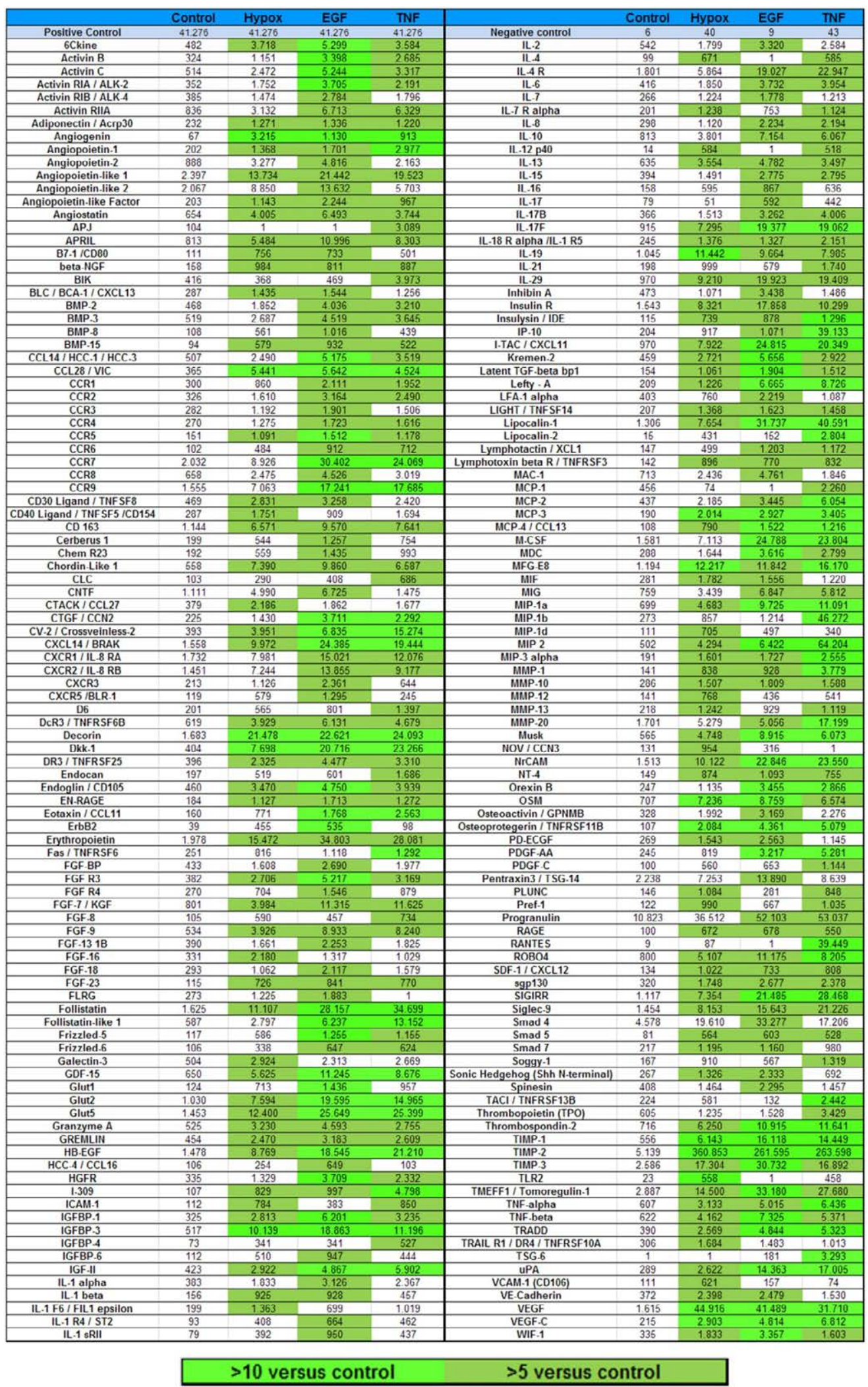

Cells were either cultured under standard conditions (Ctrl) or preconditioned by incubation in a hypoxic environment ( $\mathrm{Hyp} ; 0.5 \% \mathrm{O}_{2}$ ), in the presence of EGF (10 ng/ $\mathrm{ml}$ ) or TNFa $(10 \mathrm{ng} / \mathrm{ml})$ for $48 \mathrm{~h}$. Hereafter, expression of 507 proteins was measured in the cell supernatant by a commercially available protein array. Heatmap displays proteins enhanced at least $>5$-fold versus control and arbitrary unit of stimulation $>500$.

Figure 4: Heat map of differentially expressed proteins after preconditioning 
e.g. in acute kidney injury [20] or cardiovascular diseases [2]. Herein, paracrine factors secreted by transplanted MSCs have been reported to be largely responsible for neovascularization and tissue regeneration [21]. Their ability to express and release these factors has been analysed in many in vitro studies, including various in vitro pretreatments $[5,6,9]$. Cell therapy heavily relies on the strong capability of the transplanted cells to deliver regeneration promoting factors to promote angiogenesis, wound repair, and tissue regeneration. It is also well known that pretreated MSCs hold an improvement for organ engraftment and for enhanced in vivo cell retention after transplantation compared to untreated cells.

Several strategies have been explored how to enhance the secretion of paracrine factors and, therefore, the regenerative potential of MSCs (or their $\mathrm{CM}$ ) before cell transplantation, including gene modification [22], preincubation with drugs [23] or low-dose lipopolysaccharide [24], growth factors [3,4], cytokines [21], cell-cell-interactions [25], or culture in hypoxia $[5,6]$. Nevertheless, the secretome of ASCs after different preconditioning regimens has yet not been investigated in a comprehensive manner. Thus, further studies in vitro and in vivo are required to fully unfold ASCs' paracrine secretome and to optimize the cells as an effective tool for cell therapeutical approaches. Advances in high-throughput technologies, protein microarrays, and bioinformatics have already facilitated analysis of the secretome and will continue to aid in identification of soluble factors released by stem cells under different conditions [9].

In this study we focussed on the comparison of different preconditioning regimens on cultures of ASCs. First, we analysed gene expression levels of seven factors treated with three different preconditioning regimens. Hereafter, we then completed the analysis with a commercially available human protein array. Our investigation illustrates that the release of factors into the supernatant can effectively be enhanced by all three regimens, although we achieved an individual pattern of secreted growth factors and cytokines (Figure 4 and Table S1). Interestingly, we could show that the short-term preconditioning with EGF yielded the strongest release of proteins, whereas all three regimens induced an increase of many factors. In contrast, mRNA expression of the seven investigated genes remained relatively unchanged via preconditioning with EGF in comparison the control.

Several recently published studies have shown that preconditioning by hypoxia strongly enhances the regenerative potential of MSCs $[4,26,27]$. Preconditioning by hypoxia was described to stimulate the secretion of growth factors, cytokines and other proteins and the release of microvesicles with mRNA/miRNA from MSCs (and ASCs) [28]. It was shown that hypoxic preconditioning is likewise able to enhance the angiogenic potential of human ASCs [23] and to improve cell survival in both in vitro and in vivo studies [5,6]. The positive effect of hypoxic preconditioned transplanted human ASCs has also been shown in a rat model of ischemic acute kidney injury [26]. In this study, vascularization, apoptosis, histological injury and levels of serum creatinine and blood urea nitrogen were significantly improved in the preconditioned transplanted group compared with the control groups [26]

In addition to hypoxia, several other factors can stimulate ASCs in culture. To date, however, methods for in vitro pretreatment or preconditioning, eventually by a combination of factors, have not been fully optimized to improve ASC- or their conditioned medium-based therapies. Indeed, also the growth factor EGF and the proinflammatory mediator TNF $\alpha$ have been shown to enhance paracrine and autocrine functions of MSCs [29-32]. EGF was shown to facilitate in vitro expansion of MSCs without altering the multipotency of the cells $[31,32]$.

Understanding the mechanisms by which ASCs secrete growth factors and cytokines could be an important step to further enhance their regeneration promoting effects [33]. Whereas EGF and TNFa bind to specific receptors, the effects of hypoxia are mainly mediated through a reduced hydroxylation of hypoxia-inducible transcription factors (HIF) resulting in stabilization of the factors and translocation to the nucleus [27]. Nevertheless, hypoxic incubation has also been shown to induce phosphorylation of surface receptors and, therefore, induce several intracellular signaling pathways [27]. On the other hand, also the downstream signaling mechanisms following EGF or TNFa receptor binding initiate several signal transduction cascades (e.g. ERK, p38, and JNK MAP Kinases, IP, AKT) resulting in proliferation, transcription and protein synthesis. Due to the involvement of these various signaling pathways in all of the three pretreatment regimens further studies are needed to determine the mechanisms ultimately responsible for ASCs enhanced secretion of proteins.

Data from Tamama et al. also suggest that the molecular machinery underlying MSCs' strong paracrine capability lies downstream of EGF receptor (EGFR) signaling. The expression of a functional EGFR and the signal transduction of ASCs have been shown by previous data from our group [34]. Whereas the influence of EGF on MSCs proliferation, multipotency, and cell motility and migration are well described $[31,32,34]$, no comprehensive study investigating the effect of EGF on the release of factors has been published. The effect of EGF has only been shown to enhance the release of factors like VEGF, hepatocyte growth factor, HB-EGF, and interleukin-6 and -11 [35]. These data strongly suggest that EGF can be used for in vitro MSC expansion and for the enhancement of ASCs` paracrine capability.

TNFa-pretreated ASCs have been shown to release various proteins, including cytokines, extracellular matrix, proteases, and protease inhibitors [24]. For example, treatment with TNFa has been reported to stimulate VEGF secretion in ASCs up to 1.5 fold [30]. Lee and coworkers identified 118 enhanced secreted proteins (mainly cytokines, chemokines, and proteases) by liquid chromatography coupled with tandem mass spectrometry in ASC-conditioned media upon TNFa pretreatment [24]. In addition, conditioned medium derived from TNFa pretreated ASCs has been shown to accelerate wound healing and angiogenesis in vivo [29].

The present study clearly showed that ASCs' release of soluble factors can be largely enhanced by different preconditioning regimens. Nevertheless, results from qPCR analysis were not congruent to results of the protein array, maybe due to the chosen endpoint analysis after 48 hours for both assays. It is possible that mRNA is already degraded at this time point and additional experiments at an earlier time point are needed.

In conclusion, all three different preconditioning regimens induced a wide variety of proteins, yet short-term pretreatment with EGF induced the highest quantity of proteins, and, therefore, appears to be the best preconditioning regime for cell therapeutic approaches.

\section{Acknowledgement}

Dr. Gerhard Sattler, Rosenparkklinik Darmstadt, Germany, is thanked for supply with liposuctionaspirates. This work was supported by the Adolf MesserStiftung, Bad Soden, Germany.

\section{References}

1. Cruz FF, Rocco PR (2015) Hypoxic preconditioning enhances mesenchymal stromal cell lung repair capacity. Stem Cell Res Ther 6: 130. [PubMed] 
2. Trivedi P, Tray N, Nguyen T, Nigam N, Gallicano GI (2010) Mesenchymal stem cell therapy for treatment of cardiovascular disease: helping people sooner or later. Stem Cells Dev 19: 1109-1120. [PubMed]

3. Hahn J, Cho H, Kang H, Kim T, Kim M, et al. (2008) Pre-treatment of mesenchymal stem cells with a combination of growth factors enhances gap junction formation, cytoprotective effect on cardiomyocytes, and therapeutic efficacy for myocardial infarction. J Am Coll Cardiol 51: 933-943. [PubMed]

4. Rota C, Imberti B, Pozzobon M, Piccoli M, De Coppi P, et al. (2012) Human amniotic fluid stem cell preconditioning improves their regenerative potential. Stem Cells Dev 21: 1911-1923. [PubMed]

5. Stubbs SL, Hsiao ST, Peshavariya HM, Lim SY, Dusting GJ, et al. (2012) Hypoxic preconditioning enhances survival of human adipose-derived stem cells and conditions endothelial cells in vitro. Stem Cells Dev 21: 1887-1896. [PubMed]

6. Beegle J, Lakatos K, Kalomoiris S, Stewart H, Isseroff RR, et al. (2015) Hypoxic preconditioning of mesenchymal stromal cells induces metabolic changes, enhances survival, and promotes cell retention in vivo. Stem Cells 33: 18181828. [PubMed]

7. Da Silva Meirelles L, Chagastelles PC, Nardi NB (2006) Mesenchymal stem cells reside in virtually all post-natal organs and tissues. J Cell Sci 119: 22042213. [PubMed]

8. Baer PC, Geiger H (2012) Adipose-derived mesenchymal stromal/stem cells: tissue localization, characterization, and heterogeneity. Stem Cells Int 2012: 812693. [PubMed]

9. Tran C, Damaser MS (2014) Stem cells as drug delivery methods: application of stem cell secretome for regeneration. Adv Drug Deliv Rev 82-83: 1-11. [PubMed]

10. Caplan Al (2008) All MSCs Are Pericytes? Cell Stem Cell 3: 229-230. [PubMed]

11. Hsiao ST, Asgari A, Lokmic Z, Sinclair R, Dusting GJ, et al. (2012) Comparative analysis of paracrine factor expression in human adult mesenchymal stem cells derived from bone marrow, adipose, and dermal tissue. Stem Cells Dev 21: 2189-2203. [PubMed]

12. Brzoska M, Geiger H, Gauer S, Baer PC (2005) Epithelial differentiation of human adipose tissue-derived adult stem cells. Biochemical and Biophysical Research Communications 330: 142-150. [PubMed]

13. Baer PC, Kuçi S, Krause M, Kuçi Z, Zielen S, et al. (2013) Comprehensive phenotypic characterization of human adipose-derived stromal/stem cells and their subsets by a high throughput technology. Stem Cells Dev 22: 330-339. [PubMed]

14. Griesche N, Bereiter-Hahn J, Geiger H, Schubert R, Baer PC (2012) During epithelial differentiation of human adipose-derived stromal/stem cells, expression of zonula occludens protein-1 is induced by a combination of retinoic acid, activin-A and bone morphogenetic protein-7. Cytotherapy 14: 6169. [PubMed]

15. Griesche N, Luttmann W, Luttmann A, Stammermann T, Geiger H, et al. (2010) A simple modification of the separation method reduces heterogeneity of adipose-derived stem cells. Cells Tissues Organs 192: 106-115. [PubMed]

16. Baer PC, Griesche N, Luttmann W, Schubert R, Luttmann A, et al. (2010) Human adipose-derived mesenchymal stem cells in vitro: evaluation of an optimal expansion medium preserving stemness. Cytotherapy 12: 96-106. [PubMed]

17. Chomczynski $P$, Sacchi N (2006) The single-step method of RNA isolation by acid guanidinium thiocyanate - phenol - chloroform extraction : twentysomething years on. Nat Protoc 1: 581-585. [PubMed]

18. Pfaffl MW (2001) A new mathematical model for relative quantification in realtime RT-PCR. Nucleic Acids Res 29: e45. [PubMed]

19. Phinney DG, Prockop DJ (2007) Concise review: mesenchymal stem/ multipotent stromal cells: the state of transdifferentiation and modes of tissue repair-current views. Stem Cells 25: 2896-2902. [PubMed]

20. Morigi M, Introna M, Imberti B, Corna D, Abbate M, et al. (2008) Human bone marrow mesenchymal stem cells accelerate recovery of acute renal injury and prolong survival in mice. Stem Cells 26: 2075-2082. [PubMed]

21. Kwon YW, Heo SC, Jeong GO, Yoon JW, Mo WM, et al. (2013) Tumor necrosis factor-a-activated mesenchymal stem cells promote endothelial progenitor cel homing and angiogenesis. Biochim Biophys Acta 1832: 2136-2144. [PubMed]

22. Gheisari Y, Azadmanesh K, Ahmadbeigi N, Nassiri SM, Golestaneh AF, et al. (2012) Genetic modification of mesenchymal stem cells to overexpress CXCR4 and CXCR7 does not improve the homing and therapeutic potentials of these cells in experimental acute kidney injury. Stem Cells Dev 21: 2969-2980. [PubMed]

23. Carrière A, Ebrahimian TG, Dehez S, Aug N, Joffre C, et al. (2009) Preconditioning by mitochondrial reactive oxygen species improves the proangiogenic potential of adipose-derived cells-based therapy. Arterioscler Thromb Vasc Biol 29: 1093-1099. [PubMed]

24. Lee MJ, Kim J, Kim MY, Bae YS, Ryu SH, et al. (2010) Proteomic analysis of tumor necrosis factor-alpha-induced secretome of human adipose tissuederived mesenchymal stem cells. J Proteome Res 9: 1754-1762. [PubMed]

25. Frith JE, Thomson B, Genever PG (2010) Dynamic three-dimensional culture methods enhance mesenchymal stem cell properties and increase therapeutic potential. Tissue Eng Part C Methods 16: 735-749. [PubMed]

26. Zhang W, Liu L, Huo Y, Yang Y, Wang Y (2014) Hypoxia-Pretreated Human MSCs Attenuate Acute Kidney Injury through Enhanced Angiogenic and Antioxidative Capacities. Biomed Res Int 2014: 462472. [PubMed]

27. Kang S, Kim SM, Sung JH (2014) Cellular and molecular stimulation of adiposederived stem cells under hypoxia. Cell Biol Int 38: 553-562. [PubMed]

28. Bruno S, Grange C, Collino F, Deregibus MC, Cantaluppi V, et al. (2012) Microvesicles derived from mesenchymal stem cells enhance survival in a lethal model of acute kidney injury. PLoS One 7: e33115. [PubMed]

29. Heo SC, Jeon ES, Lee IH, Kim HS, Kim MB, et al. (2011) Tumor necrosis factor alpha-activated human adipose tissue-derived mesenchymal stem cells accelerate cutaneous wound healing through paracrine mechanisms. J Invest Dermatol 131: 1559-1567. [PubMed]

30. Wang M, Crisostomo PR, Herring C, Meldrum KK, Meldrum DR (2006) Human progenitor cells from bone marrow or adipose tissue produce VEGF, HGF, and IGF-I in response to TNF by a p38 MAPK-dependent mechanism. Am J Physio Regul Integr Comp Physiol 291: R880-R884. [PubMed]

31. Tamama K, Fan VH, Griffith LG, Blair HC, Wells A (2006) Epiderma growth factor as a candidate for ex vivo expansion of bone marrow-derived mesenchymal stem cells. Stem Cells 24: 686-695. [PubMed]

32. Tamama K, Kawasaki H, Wells A (2010) Epidermal growth factor (EGF) treatment on multipotential stromal cells (MSCs). Possible enhancement of therapeutic potential of MSC. J Biomed Biotechnol 2010: 795385. [PubMed]

33. Crisostomo PR, Wang Y, Markel TA, Wang M, Lahm T, et al. (2008) Human mesenchymal stem cells stimulated by TNF-alpha, LPS, or hypoxia produce growth factors by an NF kappa B- but not JNK-dependent mechanism. Am J Physiol Cell Physiol 294: C675-682. [PubMed]

34. Baer PC, Schubert R, Bereiter-Hahn J, Ploesser M, Geiger H (2009) Expression of a functional epidermal growth factor receptor on human adipose-derived mesenchymal stem cells and its signaling mechanism. Eur J Cell Biol 88: 273283. [PubMed]

35. Kerpedjieva SS, Kim DS, Barbeau DJ, Tamama K (2012) EGFR ligands drive multipotential stromal cells to produce multiple growth factors and cytokines via early growth response-1. Stem Cells Dev 21: 2541-2551. [PubMed] 